\title{
Phenomenological Constraints on the Transport Properties of QCD Matter with Data-Driven Model Averaging
}

D. Everett $\odot,{ }^{1}$ W. Ke, ${ }^{2,3}$ J.-F. Paquet, ${ }^{4}$ G. Vujanovic, ${ }^{5}$ S. A. Bass, ${ }^{4}$ L. Du, ${ }^{1}$ C. Gale, ${ }^{6}$ M. Heffernan, ${ }^{6}$ U. Heinz, ${ }^{1}$ D. Liyanage, ${ }^{1}$ M. Luzum, ${ }^{7}$ A. Majumder, ${ }^{5}$ M. McNelis, ${ }^{1}$ C. Shen,${ }^{5,8}$ Y. Xu, ${ }^{4}$ A. Angerami, ${ }^{9}$ S. Cao, ${ }^{5}$ Y. Chen, ${ }^{10,11}$ J. Coleman, ${ }^{12}$ L. Cunqueiro, ${ }^{13,14}$ T. Dai, ${ }^{4}$ R. Ehlers, ${ }^{13,14}$ H. Elfner, ${ }^{15,16,17}$ W. Fan, ${ }^{4}$ R. J. Fries, ${ }^{18,19}$ F. Garza, ${ }^{18,19}$ Y. He,${ }^{20}$ B. V. Jacak, ${ }^{2,3}$ P. M. Jacobs, ${ }^{2,3}$ S. Jeon, ${ }^{6}$ B. Kim, ${ }^{18,19}$ M. Kordell II, ${ }^{18,19}$ A. Kumar, ${ }^{5}$ S. Mak, ${ }^{12}$ J. Mulligan, ${ }^{2,3}$ C. Nattrass, ${ }^{13}$

D. Oliinychenko, ${ }^{3}$ C. Park, ${ }^{6}$ J. H. Putschke, ${ }^{5}$ G. Roland, ${ }^{10,11}$ B. Schenke, ${ }^{21}$ L. Schwiebert, ${ }^{22}$ A. Silva, ${ }^{13}$ C. Sirimanna, ${ }^{5}$ R. A. Soltz, ${ }^{5,9}$ Y. Tachibana, ${ }^{5}$ X.-N. Wang, ${ }^{20,2,3}$ and R. L. Wolpert ${ }^{12}$

(JETSCAPE Collaboration)

\author{
${ }^{1}$ Department of Physics, The Ohio State University, Columbus, Ohio 43210, USA \\ ${ }^{2}$ Department of Physics, University of California, Berkeley, California 94270, USA \\ ${ }^{3}$ Nuclear Science Division, Lawrence Berkeley National Laboratory, Berkeley, California 94270, USA \\ ${ }^{4}$ Department of Physics, Duke University, Durham, North Carolina 27708, USA \\ ${ }^{5}$ Department of Physics and Astronomy, Wayne State University, Detroit, Michigan 48201, USA \\ ${ }^{6}$ Department of Physics, McGill University, Montréal, Quebec City H3A 2T8, Canada \\ ${ }^{7}$ Instituto de Fìsica, Universidade de São Paulo, C.P. 66318, 05315-970 São Paulo, SP, Brazil \\ ${ }^{8}$ RIKEN BNL Research Center, Brookhaven National Laboratory, Upton, New York 11973, USA \\ ${ }^{9}$ Lawrence Livermore National Laboratory, Livermore, California 94550, USA \\ ${ }^{10}$ Laboratory for Nuclear Science, Massachusetts Institute of Technology, Cambridge, Massachusetts 02139, USA \\ ${ }^{11}$ Department of Physics, Massachusetts Institute of Technology, Cambridge, Massachusetts 02139, USA \\ ${ }^{12}$ Department of Statistical Science, Duke University, Durham, North Carolina 27708, USA \\ ${ }^{13}$ Department of Physics and Astronomy, University of Tennessee, Knoxville, Tennessee 37996, USA \\ ${ }^{14}$ Physics Division, Oak Ridge National Laboratory, Oak Ridge, Tennessee 37830, USA \\ ${ }^{15}$ GSI Helmholtzzentrum für Schwerionenforschung, 64291 Darmstadt, Germany \\ ${ }^{16}$ Institute for Theoretical Physics, Goethe University, 60438 Frankfurt am Main, Germany \\ ${ }^{17}$ Frankfurt Institute for Advanced Studies, 60438 Frankfurt am Main, Germany \\ ${ }^{18}$ Cyclotron Institute, Texas A\&M University, College Station, Texas 77843, USA \\ ${ }^{19}$ Department of Physics and Astronomy, Texas A\&M University, College Station, Texas 77843, USA \\ ${ }^{20}$ Key Laboratory of Quark and Lepton Physics (MOE) and Institute of Particle Physics, Central China Normal University, \\ Wuhan 430079, China \\ ${ }^{21}$ Physics Department, Brookhaven National Laboratory, Upton, New York 11973, USA \\ ${ }^{22}$ Department of Computer Science, Wayne State University, Detroit, Michigan 48202, USA
}

(Received 2 November 2020; revised 5 March 2021; accepted 27 May 2021; published 17 June 2021)

Using combined data from the Relativistic Heavy Ion and Large Hadron Colliders, we constrain the shear and bulk viscosities of quark-gluon plasma (QGP) at temperatures of $\sim 150-350 \mathrm{MeV}$. We use Bayesian inference to translate experimental and theoretical uncertainties into probabilistic constraints for the viscosities. With Bayesian model averaging we propagate an estimate of the model uncertainty generated by the transition from hydrodynamics to hadron transport in the plasma's final evolution stage, providing the most reliable phenomenological constraints to date on the QGP viscosities.

DOI: 10.1103/PhysRevLett.126.242301

Published by the American Physical Society under the terms of the Creative Commons Attribution 4.0 International license. Further distribution of this work must maintain attribution to the author(s) and the published article's title, journal citation, and DOI. Funded by SCOAP.
Introduction.-Ultrarelativistic collisions of heavy nuclei provide an experimental avenue to produce quarkgluon plasma (QGP), a short-lived state of deconfined hot and dense nuclear matter [1-7]. Quark-gluon plasma produced in heavy-ion collisions is a strongly coupled fluid [8] that can be characterized by its macroscopic properties such as its equation of state and transport coefficients. These macroscopic characteristics encode the underlying 
microscopic interactions, described by quantum chromodynamics (QCD), among the fluid's constituents.

Understanding the different phases of QCD matter remains an area of topical interest in nuclear physics. The equation of state of deconfined nuclear matter with no net baryon density has been known from first principles for more than a decade, by computing the QCD equilibrium partition function numerically on a space-time lattice $[9,10]$. Calculating the transport coefficients of such nuclear plasma is a continuing challenge [11], however: significant numerical and theoretical uncertainties currently limit the evaluation of the relevant energy-momentum tensor correlators through lattice techniques [12]. Moreover, the strongly coupled microscopic dynamics of the plasma in the experimentally accessible temperature range, $\sim 150-350 \mathrm{MeV}$, precludes description via perturbative approaches $[13,14]$.

In this work, we use large sets of hadronic measurements from the Relativistic Heavy Ion Collider (RHIC) and the Large Hadron Collider (LHC) to constrain the temperature dependence of two QGP transport coefficients: the shear and bulk viscosities. We make the case that reliable constraints on these viscosities can only be obtained by large scale model-to-data comparisons which account methodically for theoretical uncertainties. Building on previous studies [15-21] and Bayesian inference literature [22,23], we put forward a general and systematically improvable framework to constrain the viscosities of the QGP from current and future measurements in heavy ion collisions.

The QGP viscosities can be constrained as a consequence of their measurable effects on the momentum distribution of hadrons produced in heavy-ion collisions [6,24-26]. Qualitatively, a large bulk viscosity tends to isotropically reduce the momenta of hadrons, while shear viscosity decreases their azimuthal momentum asymmetry. Despite these characteristic effects, constraining viscosities from measurements is a challenging undertaking. The hydrodynamic expansion of the deconfined plasma represents one of many stages of heavy ion collisions, with others regimes of many-body nuclear dynamics preceding and succeeding this fluid phase. The hydrodynamic evolution itself has intricate flow velocity and temperature profiles, which fluctuate from collision to collision; this leads to considerable difficulties in factorizing the effect of the shear and bulk viscosities using hadronic measurements.

To meaningfully constrain QGP viscosities from collider measurements, multiple stages of the collision must therefore be precisely modeled and the resulting predictions compared with large and diverse sets of experimental data. Theoretical uncertainties in every stage of the collision can significantly affect estimation of QGP viscosities. Yet model-data inference may be the only methodology currently capable of estimating the transport coefficients of strongly coupled QGP with quantified uncertainties [11]. In this context, the framework we put forward provides a path to reliably constrain these viscosities, accounting for both experimental and theoretical uncertainties. As a major step forward, this contribution includes for the first time a methodology to quantify and incorporate the irreducible modeling ambiguities in the transition from a fluid dynamical description of the plasma to a microscopic kinetic evolution of the late hadronic stage.

Modeling heavy-ion collisions.-In a collision between two heavy ions, a fraction of their large kinetic energy is converted into a color-deconfined form of excited nuclear matter. The creation and subsequent evolution of this newly created matter span several different successive many-body regimes of QCD, which can be described with a multistage model. We provide a brief summary of this model, referring for more details to the longer companion paper [27]. The energy deposition at midrapidity during the primary impact is described with the $T_{R}$ ENTO ansatz [28,29]; this model accounts for the varying degree of overlap between the colliding nuclei, and for fluctuations in the positions of their nucleons and the amount of energy deposited in each nucleon-nucleon collision. The energy-momentum tensor describing this early, extremely dense stage of the collision is subsequently evolved for a brief period of $\mathcal{O}(0.1-1) \mathrm{fm} / c$ as an ensemble of free-streaming massless particles [30-32]; for sufficiently weakly coupled systems, it is well established [33-37] that free-streaming approximates well the early prehydrodynamic evolution stage. At the end of the free-streaming stage the energy-momentum tensor is matched to $(2+1) \mathrm{D}$ dissipative hydrodynamics. In this matching process, space-momentum correlations that developed during the free-streaming stage manifest themselves as nonzero initial flow velocity and viscous stress profiles for the subsequent hydrodynamic evolution. The second-order dissipative relativistic fluid dynamic stage [38-41] describes the evolution of the quark-gluon plasma fluid and forms the core of the simulation. Its most important ingredients are a first-principles equation of state from lattice QCD [10,42,43] and two parametrized [27] first-order transport coefficients: the specific shear and bulk viscosities. When the fluid has cooled to the pseudocritical temperature $T_{c}$, the color-charged QGP constituents reorganize into color-neutral hadrons. This color neutralization causes a rapid increase in mean free path, quickly leading to a breakdown of local thermal equilibrium; this requires transitioning [44-46] from a macroscopic fluid dynamical picture to hadronic kinetic transport [47-49].

A key aspect of our description of heavy-ion collisions is its unprecedented flexibility. It models a multitude of dynamical details that are expected to be phenomenologically important, but are not theoretically well constrained. For example, the initialization time of hydrodynamics is allowed to vary with the amount of energy deposited in the collision, since larger energy densities result in shorter mean free paths and faster hydrodynamization of the fluid [50]. Combined with the flexibility of the $T_{R}$ ENTO ansatz 
for the energy deposition followed by free-streaming, this offers a wide range of scenarios for the prehydrodynamic collision stage. For the shear and bulk viscosities we employ more general parametrizations than in previous studies [20]. For example, $\zeta / s(T)$ is assumed to have a peak in the deconfinement region as in Ref. [20], but the profile around the peak is allowed to be asymmetric in temperature, while a wider range of values for the width, maximum, and position of this peak [27] are explored. A second-order transport coefficient - the shear relaxation time-is also varied in order to quantify its effects on viscosity constraints.

Most importantly, we extend the Bayesian inference framework $[19,20]$ to include the uncertainties arising from a set of possible models. The transition from hydrodynamics to hadronic kinetics for the final evolution stage (called particlization) is an ill-defined problem [51]. Indeed, kinetic theory requires initial conditions for the entire hadronic phase-space distribution, whereas a hydrodynamical evolution provides (in our case) information solely about the 10 hydrodynamic moments of the hadronic distribution functions, summed over all hadron species contributing to the energy-momentum tensor $T^{\mu \nu}$. Without additional information about the microscopic dynamics that underlies the fluid dynamical evolution, an irreducible uncertainty in the hadron phase-space distribution at the fluid-hadron switching surface is unavoidable. Three commonly used prescriptions (labeled by $\mathcal{M}_{i}, i=1,2,3$ ) mapping $T^{\mu \nu}$ components onto the hadronic phase-space distributions were selected: the 14-moment Grad [52-56] approximation, relativistic Chapman-Enskog series in the relaxation-time approximation ("RTA Chapman-Enskog") [57,58], and Pratt-Torrieri-Bernhard [42,59] models. By quantifying, for the first time, the effect of this discrete model ambiguity on the posterior probability distributions, we obtain more reliable constraints on the QGP viscosities than achieved before.

Data selection.-Our model is calibrated using measurements from the LHC and RHIC. For $\mathrm{Pb}-\mathrm{Pb}$ collisions at $\sqrt{s_{\mathrm{NN}}}=2.76 \mathrm{TeV}$ we use (i) the average number $d N / d y$ of pions, kaons, and protons produced in the collisions, along with their mean transverse momentum $\left\langle p_{T}\right\rangle$ [60]; (ii) the total number of charged hadrons $d N_{\mathrm{ch}} / d \eta$ [61], along with the fluctuation $\delta p_{T} /\left\langle p_{T}\right\rangle$ of the average momentum [62]; (iii) the total transverse energy of hadrons $d E_{T} / d \eta$ [63]; and (iv) the momentum anisotropies $v_{n}\{2\}$, $n=2,3,4$, of charged hadrons in the plane transverse to the collision axis, as measured through two-particle correlations [64]. Furthermore, simulated collisions of Au nuclei with $\sqrt{s_{\mathrm{NN}}}=0.2 \mathrm{TeV}$ are compared with a smaller subset of RHIC measurements: $d N / d y$ and $\left\langle p_{T}\right\rangle$ of pions and kaons [65], as well as the momentum anisotropies $v_{2 / 3}\{2\}$ of charged hadrons $[66,67]$. Note that proton observables are only included at the LHC.

Data-driven constraints on the QGP viscosities.Experimental measurements are effectively probability distributions, which we assume to be normally distributed around their means [68]. The model calculations have statistical uncertainties as well due to simulating a finite number of minimum-bias heavy ion collisions. Accordingly, they are modeled as normal probability distributions, with estimated means and standard deviations.

Let $\mathbf{y}_{\exp }$ denote the full set of experimental measurements and $\boldsymbol{x}$ represent all the model parameters, including those governing the temperature dependences of the QGP viscosities. Discrete model choices are labeled with the index $i$; in our case, this index distinguishes between different particlization models $\mathcal{M}_{i}$. The model and experimental probability distributions are connected by the "likelihood," the probability of the model $\mathcal{M}_{i}$ being consistent with the data $\mathbf{y}_{\exp }$ at a given value of its parameters $\boldsymbol{x}$ :

$$
\mathcal{P}^{(i)}\left(\mathbf{y}_{\exp } \mid \boldsymbol{x}\right)=\frac{\exp \left[-\frac{1}{2}\left(\Delta \mathbf{y}_{\boldsymbol{x}}^{(i)}\right)^{T} \Sigma_{(i)}^{-1}(\boldsymbol{x}) \Delta \mathbf{y}_{\boldsymbol{x}}^{(i)}\right]}{\sqrt{(2 \pi)^{n} \operatorname{det}\left[\Sigma_{(i)}(\boldsymbol{x})\right]}} .
$$

Here $\Delta \mathbf{y}_{\boldsymbol{x}}^{(i)} \equiv \mathbf{y}_{\boldsymbol{x}}^{(i)}-\mathbf{y}_{\exp }$ is the discrepancy between the measurements $\mathbf{y}_{\exp }$ and their predictions $\mathbf{y}_{x}^{(i)}$ by model $i$ with the parameters $\boldsymbol{x} ; n$ is the number of data points (length of $\mathbf{y}_{\text {exp }}$ ); and $\Sigma_{(i)}(\mathbf{x})$ is a covariance matrix encoding both experimental and model uncertainties and their correlations. Experimental contributions to $\Sigma_{(i)}(\boldsymbol{x})$ are assumed to be diagonal. Although systematic uncertainties generally introduce nondiagonal terms, we neglect them at the moment since we have insufficient information from current data.

Constraints on the viscosity are given by the probability $\mathcal{P}^{(i)}\left(\boldsymbol{x} \mid \mathbf{y}_{\text {exp }}\right)$ (called "posterior") for the parameters $\boldsymbol{x}$ given a set of measurements $\mathbf{y}_{\exp }$, which according to Bayes' theorem is

$$
\mathcal{P}^{(i)}\left(\boldsymbol{x} \mid \mathbf{y}_{\exp }\right)=\frac{\mathcal{P}^{(i)}\left(\mathbf{y}_{\exp } \mid \boldsymbol{x}\right) \mathcal{P}(\boldsymbol{x})}{\mathcal{P}^{(i)}\left(\mathbf{y}_{\text {exp }}\right)} .
$$

The shape of the posterior's dependence on $\boldsymbol{x}$ is controlled by two factors: (i) the likelihood $\mathcal{P}^{(i)}\left(\mathbf{y}_{\exp } \mid \boldsymbol{x}\right)$ which accounts for the information provided by the measurements $\mathbf{y}_{\exp }$ and their uncertainties, and (ii) the prior probability $\mathcal{P}(\boldsymbol{x})$ that is assigned to the parameters before taking the current dataset into account. The prior $\mathcal{P}(\boldsymbol{x})$ reflects the combined theoretical and experimental prior knowledge about the parameters $\boldsymbol{x}$; for example, it allows theoretical constraints on certain parameters, such as positivity and causality, to be enforced. The normalization of the posterior is controlled by the "Bayesian model evidence" $\mathcal{P}^{(i)}\left(\mathbf{y}_{\text {exp }}\right)=\int d \boldsymbol{x} \mathcal{P}^{(i)}\left(\mathbf{y}_{\text {exp }} \mid \boldsymbol{x}\right) \mathcal{P}(\boldsymbol{x})$, which describes the validity of model $i$ given the data $\mathbf{y}_{\text {exp }}$; as will be 
discussed below, it can be used to discriminate between different models.

Equation (2) shows that the parameter constraints encoded in the posterior $\mathcal{P}^{(i)}\left(\boldsymbol{x} \mid \mathbf{y}_{\text {exp }}\right)$ can be improved in multiple ways. First, this can be achieved with better external constraints on the model's parameters $\boldsymbol{x}$, reflected in more realistic prior distribution $\mathcal{P}(\boldsymbol{x})$. Second, new or more precise experimental data can tighten the likelihood $\mathcal{P}^{(i)}\left(\mathbf{y}_{\exp } \mid \boldsymbol{x}\right)$. Finally, theoretical progress on the model and better quantification of the model's uncertainties lead to more reliable constraints through the likelihood as well. The ability to include both theoretical and experimental progress consistently and equitably in the extraction of new knowledge is a key feature of Bayesian inference.

The temperature dependence of the QGP viscosities favored by the RHIC and LHC data are given by evaluating the posterior (2) and marginalizing over all parameters except the viscosities. Figure 1 shows the $90 \%$ credibility ranges (outlined by colored lines) for the marginalized posterior of the three particlization models studied here. The high-credibility ranges for the different particlization models show similar qualitative features; however they differ significantly in detail, especially in the lowtemperature region between 150 and $250 \mathrm{MeV}$ where the likelihood tightens. Importantly, at high temperature, the posteriors are close to the $90 \%$ credibility ranges of the prior (gray shaded region): this strongly suggests that measurements used in this work do not constrain the viscosities significantly for temperatures $\gtrsim 250 \mathrm{MeV}$.

Note that previous studies [20] employed rather narrow priors [choice of $\mathcal{P}(\boldsymbol{x})$ for $\eta / s$ and $\zeta / s$ ] at high temperatures. Our work shows that the heavy-ion data we use are very weakly informative for $T \gtrsim 200 \mathrm{MeV}$. Hightemperature constraints obtained in Ref. [20] originated from prior assumptions [27], highlighting the importance of carefully exploring the sensitivity to prior assumptions.

More generally, it is important to emphasize that narrower posterior credible intervals are not automatically more accurate. The inclusion of previously neglected
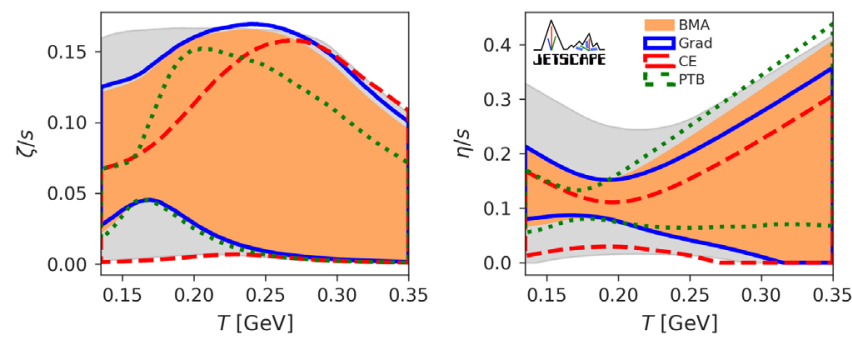

FIG. 1. The $90 \%$ credible intervals for the prior (gray), the posteriors of the Grad (blue), Chapman-Enskog (red), and PrattTorrieri-Bernhard (green) models, and their Bayesian model average (orange) for the specific bulk (left) and shear (right) viscosities of QGP. model uncertainties generally leads to wider, yet more reliable, credible intervals.

At the moment, there is insufficient theoretical evidence to establish which model is a better description of the particlization process in heavy-ion collisions. In absence of such prior theoretical insight, we use experimental measurements to judge the quality of each particlization model. This is done by using the Bayes evidence $\mathcal{P}^{(i)}\left(\mathbf{y}_{\exp }\right)$ from Eq. (2), which corresponds to the average of the likelihood over the parameter space. Bayes evidence favors good agreement with data (high likelihood) while disfavoring model complexity [69]. The ratio of Bayes evidences is approximately 5000:2000:1 for the Grad, Pratt-Torrieri-Bernhard, and Chapman-Enskog particlization models, respectively, clearly disfavoring the Chapman-Enskog model.

The Bayesian evidence can be used as a data-driven approach to combine the results for the three particlization models into one posterior distribution [70], as defined by Bayesian model averaging [22]:

$$
\mathcal{P}_{\text {BMA }}\left(\boldsymbol{x} \mid \mathbf{y}_{\text {exp }}\right) \propto \sum_{i} \mathcal{P}^{(i)}\left(\mathbf{y}_{\exp }\right) \mathcal{P}^{(i)}\left(\boldsymbol{x} \mid \mathbf{y}_{\text {exp }}\right) .
$$

This results in the orange band in Fig. 1. Being strongly disfavored by the Bayesian evidence, the impact of the Chapman-Enskog particlization model on the Bayesian model average (3) is minor.

The level of agreement of each particlization model with a representative subset of measurements is shown in Fig. 2. The bands represent the $90 \%$ posterior predictive distributions of observables, obtained by sampling the parameter posterior $\mathcal{P}^{(i)}\left(\boldsymbol{x} \mid \mathbf{y}_{\exp }\right)$. All three particlization models show reasonable agreement with the data, giving credence to
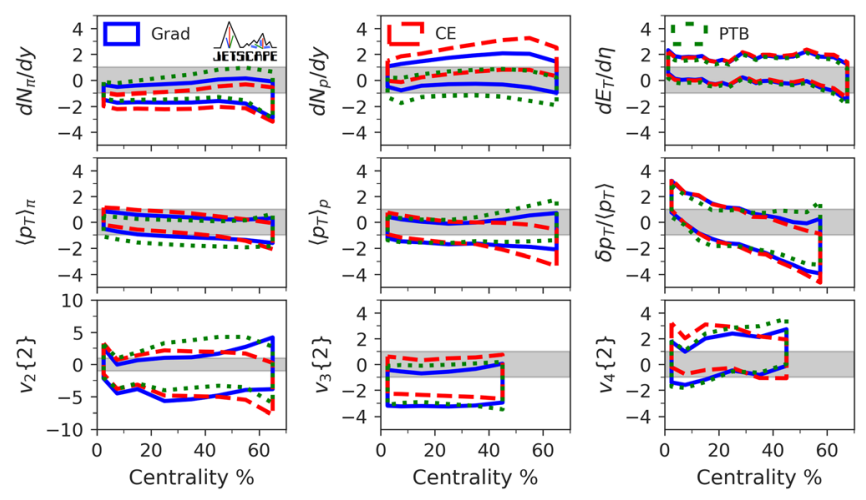

FIG. 2. The $90 \%$ credible intervals of the posterior predictive distribution of observables for $\mathrm{Pb}-\mathrm{Pb}$ collisions at the $\mathrm{LHC}$ as functions of centrality, for the Grad (blue), Chapman-Enskog (red), and Pratt-Torrieri-Bernhard (green) particlization models. Plotted is the model discrepancy in units of the experimental standard deviation $\sigma_{\text {exp }}$; the vertical axes are labeled with shorthand notation $y \equiv\left(y_{\text {model }}-y_{\exp }\right) / \sigma_{\exp }$, where $y$ stands for the observable whose model discrepancy is shown. The gray bands represent a discrepancy of one $\sigma_{\exp }$ above and below zero. 
their respective posterior estimates of the shear and bulk viscosity (and other model parameters) that were inferred from the model-to-data comparison. A closer look at Fig. 2 reveals tension with the Chapman-Enskog particlization model, which struggles at describing the pion and proton multiplicities simultaneously. This tension in the proton-topion ratio is the origin of its small Bayes evidence. In Ref. [27] we show that ignoring the proton $d N / d y$ reduces the odds against the Chapman-Enskog particlization model from 5000:1 to 5:1 relative to the Grad model; the key feature behind its failure is the form of its bulk viscous correction to the particle momentum distributions. This highlights the importance of understanding how energy and momentum are distributed across both momentum and species at particlization. We note that our choice of likelihood function, Eq. (1), assumes that probability decreases rapidly away from the mean; this can be unforgiving to tension with the data, resulting in the large ratios of Bayes evidence encountered in this work. Other forms of likelihood should be investigated in the future. Nevertheless, we believe the proton-to-pion ratio is an important observable: the averaged constraints consequently favor particlization models that can describe it well.

To emphasize the constraints provided by the experimental data, we calculate the information gain of our posteriors for the temperature dependence of the viscosities of QCD, relative to the corresponding priors, using the Kullback-Leibler divergence $\left(D_{K L}\right)$ [71]. The result is shown in Fig. 3 alongside the 90\% prior and Bayesian model averaged posteriors. While the experimental data are seen to provide significant constraints for $150 \lesssim T \lesssim$ $250 \mathrm{MeV}$ their constraining power rapidly degrades at higher temperatures. In the deconfinement region, the most likely values for $\eta / s$ are of order $0.1 ; \zeta / s$ also favors values around $0.05-0.1$ in that region, although constraints are weaker than for $\eta / s$. The small values of $\eta / s$ obtained at $T \approx 150 \mathrm{MeV}$ suggest tension with the larger values expected for a dilute gas of hadrons, such as in the SMASH model used in our simulations after particlization [72]. On the other hand, the bulk viscosity appears to be consistent [73]. Narrower priors could be used to limit the possible values of viscosity: for example, negative slopes
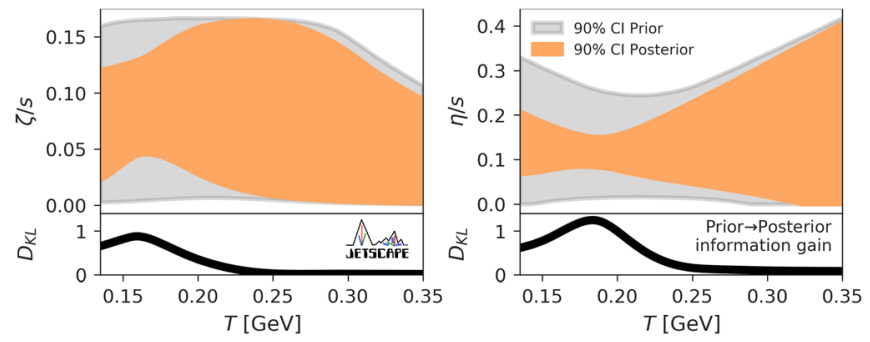

FIG. 3. $90 \%$ credible intervals for the priors (gray) and Bayesian model averaged posteriors for the specific bulk (left) and shear (right) viscosities, along with their corresponding information gain (Kullback-Leibler divergence $D_{\mathrm{KL}}$ ). for the shear viscosity at high temperature could be excluded based on theoretical guidance [20]. We elect not to do so, emphasizing instead the constraining power provided directly by measurements.

Summary.-First-principles insights into the transport properties of quark-gluon plasma are still limited for temperatures $\sim 150-350 \mathrm{MeV}$. The phenomenological constraints obtained in this work from heavy-ion measurements complement the current theoretical knowledge, supplementing a range of calculations of the shear and bulk viscosities of nuclear plasma at lower [72-74], intermediate $[12,75,76]$, and higher $[13,77]$ temperatures.

In this work, we obtained new state-of-the-art estimates for the QGP shear and bulk viscosities with more robust estimates for the uncertainties of these key transport coefficients. We introduced model averaging into Bayesian inference to include both experimental and known theoretical uncertainties in the uncertainty budget for the model parameters inferred from RHIC and LHC data. By allowing for a systematic inclusion of (i) additional measurements, (ii) model uncertainties, (iii) error correlations, and (iv) more rigorous and objective specification of model priors, the methods pioneered in this analysis for heavy-ion physics provide a clear path forward for rigorous estimations of the transport properties of the quark-gluon plasma.

We thank Jonah Bernhard, Gabriel Denicol, Scott Moreland, Scott Pratt, and Derek Teaney for useful discussions, and Richard J. Furnstahl and Xilin Zhang for their insights on Bayesian Inference and Markov Chain Monte Carlo. This work was supported in part by the National Science Foundation (NSF) within the framework of the JETSCAPE Collaboration, under Grants No. ACI1550172 (Y. C. and G. R.), No. ACI-1550221 (R. J. F., F. G., M. K., and B. K.), No. ACI-1550223 (D. E., M. M., U.H., L. D., and D. L.), ACI-1550225 (S. A. B., J.C., T. D., W. F., W. K., R. W., S. M., and Y.X.), No. ACI1550228 (J. M., B. J., P. J., X.-N. W.), and No. ACI1550300 (S. C., L.C., A. K., A. M., C. N., A. S., J.P., L. S., C. Si., R. A. S., and G. V.); it was supported in part by the NSF under Grant No. OAC-2004571 (X-SCAPE), by PHY-1516590, PHY-1812431 (R. J.F., B. K., F. G., M. K.), by PHY-2012922 (C. S.); it was supported in part by the U.S. Department of Energy, Office of Science, Office of Nuclear Physics under Grants No. DE-AC0205CH11231 (D. O., X.-N. W.), No. DE-AC52-07NA27344 (A. A., R. A. S.), No. DE-SC0013460 (S. C., A. K., A. M., C. S., and C. Si.), No. DE-SC0004286 (L. D., M. M., D. E., U.H., and D. L.), No. DE-SC0012704 (B.S. and C. S.), No. DE-FG02-92ER40713 (J. P.), and No. DE-FG0205ER41367 (T.D., W. K., J.-F.P., S. A. B., and Y.X.). The work was also supported in part by the National Science Foundation of China (NSFC) under Grants No. 11935007, No. 11861131009, and No. 11890714 (Y.H. and X.-N.W.), by the Natural Sciences and Engineering Research Council of Canada (C. G., M. H., 
S. J., C.P., and G. V.), by the Fonds de Recherche du Québec Nature et Technologies (FRQ-NT) (G. V.), by the Office of the Vice President for Research (OVPR) at Wayne State University (Y. T.), by the São Paulo Research Foundation (FAPESP) under Projects No. 2016/24029-6, No. 2017/05685-2 and No. 2018/24720-6 (M. L.), and by the University of California, Berkeley-Central China Normal University Collaboration Grant (W. K.). U. H. would like to acknowledge support by the Alexander von Humboldt Foundation through a Humboldt Research Award. Allocation of supercomputing resources (Project No. PHY180035) were obtained in part through the Extreme Science and Engineering Discovery Environment (XSEDE), which is supported by National Science Foundation Grant No. ACI-1548562. Calculation were performed in part on Stampede2 compute nodes, generously funded by the National Science Foundation (NSF) through Grant No. ACI-1134872, within the Texas Advanced Computing Center (TACC) at the University of Texas at Austin [78], and in part on the Ohio Supercomputer [79] (Project No. PAS0254). Computations were also carried out on the Wayne State Grid funded by the Wayne State OVPR, and on the supercomputer Guillimin from McGill University, managed by Calcul Québec and Compute Canada. The operation of the supercomputer Guillimin is funded by the Canada Foundation for Innovation (CFI), NanoQuébec, Réseau de Médicine Génétique Appliquée (RMGA) and FRQ-NT. Data storage was provided in part by the OSIRIS project supported by the National Science Foundation under Grant No. OAC-1541335.

[1] I. Arsene et al. (BRAHMS Collaboration), Quark gluon plasma and color glass condensate at RHIC? The perspective from the BRAHMS experiment, Nucl. Phys. A757, 1 (2005).

[2] B. B. Back et al. (PHOBOS Collaboration), The PHOBOS perspective on discoveries at RHIC, Nucl. Phys. A757, 28 (2005).

[3] J. Adams et al. (STAR Collaboration), Experimental and theoretical challenges in the search for the quark gluon plasma: The STAR Collaboration's critical assessment of the evidence from RHIC collisions, Nucl. Phys. A757, 102 (2005).

[4] K. Adcox et al. (PHENIX Collaboration), Formation of dense partonic matter in relativistic nucleus-nucleus collisions at RHIC: Experimental evaluation by the PHENIX collaboration, Nucl. Phys. A757, 184 (2005).

[5] B. Muller, J. Schukraft, and B. Wyslouch, First results from $\mathrm{Pb}+\mathrm{Pb}$ collisions at the LHC, Annu. Rev. Nucl. Part. Sci. 62, 361 (2012).

[6] U. Heinz and R. Snellings, Collective flow and viscosity in relativistic heavy-ion collisions, Annu. Rev. Nucl. Part. Sci. 63, 123 (2013).
[7] W. Busza, K. Rajagopal, and W. van der Schee, Heavy ion collisions: The big picture, and the big questions, Annu. Rev. Nucl. Part. Sci. 68, 339 (2018).

[8] E. Shuryak, Strongly coupled quark-gluon plasma in heavy ion collisions, Rev. Mod. Phys. 89, 035001 (2017).

[9] S. Borsanyi, Z. Fodor, C. Hoelbling, S. D. Katz, S. Krieg, and K. K. Szabo, Full result for the QCD equation of state with $2+1$ flavors, Phys. Lett. B 730, 99 (2014).

[10] A. Bazavov et al. (HotQCD Collaboration), Equation of state in $(2+1)$-flavor QCD, Phys. Rev. D 90, 094503 (2014).

[11] G. D. Moore, Shear viscosity in QCD and why it's hard to calculate, in Criticality in QCD and the Hadron Resonance Gas (2020), arXiv:2010.15704.

[12] A. Bazavov, F. Karsch, S. Mukherjee, and P. Petreczky (USQCD Collaboration), Hot-dense lattice QCD: USQCD whitepaper 2018, Eur. Phys. J. A 55, 194 (2019).

[13] J. Ghiglieri, G. D. Moore, and D. Teaney, QCD shear viscosity at (almost) NLO, J. High Energy Phys. 03 (2018) 179.

[14] E. Shuryak, Comments on the chemical and kinetic equilibration in heavy ion collisions, arXiv:1901.00178.

[15] H. Petersen, C. Coleman-Smith, S. A. Bass, and R. Wolpert, Constraining the initial state granularity with bulk observables in $\mathrm{Au}+\mathrm{Au}$ collisions at $\sqrt{s_{\mathrm{NN}}}=200 \mathrm{GeV}$, J. Phys. G 38, 045102 (2011).

[16] J. Novak, K. Novak, S. Pratt, J. Vredevoogd, C. E. ColemanSmith, and R. L. Wolpert, Determining fundamental properties of matter created in ultrarelativistic heavy-ion collisions, Phys. Rev. C 89, 034917 (2014).

[17] E. Sangaline and S. Pratt, Toward a deeper understanding of how experiments constrain the underlying physics of heavyion collisions, Phys. Rev. C 93, 024908 (2016).

[18] J. E. Bernhard, P. W. Marcy, C. E. Coleman-Smith, S. Huzurbazar, R. L. Wolpert, and S. A. Bass, Quantifying properties of hot and dense QCD matter through systematic model-to-data comparison, Phys. Rev. C 91, 054910 (2015).

[19] J. E. Bernhard, J. S. Moreland, S. A. Bass, J. Liu, and U. Heinz, Applying Bayesian parameter estimation to relativistic heavy-ion collisions: Simultaneous characterization of the initial state and quark-gluon plasma medium, Phys. Rev. C 94, 024907 (2016).

[20] J. E. Bernhard, J. S. Moreland, and S. A. Bass, Bayesian estimation of the specific shear and bulk viscosity of quarkgluon plasma, Nat. Phys. 15, 1113 (2019).

[21] Z. Yang and R. J. Fries, Parameterizing smooth viscous fluid dynamics with a viscous blast wave, arXiv:2007.11777.

[22] J. A Hoeting, D. Madigan, A. E. Raftery, and C. T. Volinsky, Bayesian model averaging: A tutorial, Stat. Sci. 14382 (1999), https://www.jstor.org/stable/2676803?seq=1.

[23] T. M. Fragoso, W. Bertoli, and F. Louzada, Bayesian model averaging: A systematic review and conceptual classification, Int. Stat. Rev. 86, 1 (2018).

[24] P. Romatschke and U. Romatschke, Viscosity Information from Relativistic Nuclear Collisions: How Perfect is the Fluid Observed at RHIC?, Phys. Rev. Lett. 99, 172301 (2007).

[25] H. Song and U. W. Heinz, Suppression of elliptic flow in a minimally viscous quark-gluon plasma, Phys. Lett. B 658, 279 (2008). 
[26] H. Song, S. A. Bass, U. Heinz, T. Hirano, and C. Shen, $200 \mathrm{~A} \mathrm{GeV} \mathrm{Au}+\mathrm{Au}$ Collisions Serve a Nearly Perfect Quark-Gluon Liquid, Phys. Rev. Lett. 106, 192301 (2011); Erratum, Phys. Rev. Lett. 109, 139904 (2012).

[27] D. Everett et al. (JETSCAPE Collaboration), Multi-system Bayesian constraints on the transport coefficients of QCD matter, Phys. Rev. C 103, 054904 (2021).

[28] J. S. Moreland, J. E. Bernhard, and S. A. Bass, Alternative ansatz to wounded nucleon and binary collision scaling in high-energy nuclear collisions, Phys. Rev. C 92, 011901(R) (2015).

[29] https://github.com/Duke-QCD/trento.git.

[30] J. Liu, C. Shen, and U. Heinz, Pre-equilibrium evolution effects on heavy-ion collision observables, Phys. Rev. C 91, 064906 (2015); Erratum, Phys. Rev. C Phys. Rev. C 92, 049904 (2015).

[31] W. Broniowski, W. Florkowski, M. Chojnacki, and A. Kisiel, Free-streaming approximation in early dynamics of relativistic heavy-ion collisions, Phys. Rev. C 80, 034902 (2009).

[32] https://github.com/derekeverett/freestream-milne.

[33] J. Vredevoogd and S. Pratt, Universal flow in the first stage of relativistic heavy ion collisions, Phys. Rev. C 79, 044915 (2009).

[34] L. Keegan, A. Kurkela, A. Mazeliauskas, and D. Teaney, Initial conditions for hydrodynamics from weakly coupled pre-equilibrium evolution, J. High Energy Phys. 08 (2016) 171.

[35] A. Kurkela, A. Mazeliauskas, J.-F. Paquet, S. Schlichting, and D. Teaney, Effective kinetic description of event-byevent pre-equilibrium dynamics in high-energy heavy-ion collisions, Phys. Rev. C 99, 034910 (2019).

[36] A. Kurkela, A. Mazeliauskas, J.-F. Paquet, S. Schlichting, and D. Teaney, Matching the Nonequilibrium Initial Stage of Heavy Ion Collisions to Hydrodynamics with QCD Kinetic Theory, Phys. Rev. Lett. 122, 122302 (2019).

[37] S. Schlichting and D. Teaney, The first fm/c of heavy-ion collisions, Annu. Rev. Nucl. Part. Sci. 69, 447 (2019).

[38] B. Schenke, S. Jeon, and C. Gale, $(3+1)$ D hydrodynamic simulation of relativistic heavy-ion collisions, Phys. Rev. C 82, 014903 (2010).

[39] B. Schenke, S. Jeon, and C. Gale, Elliptic and Triangular Flow in Event-By-Event $(3+1) D$ Viscous Hydrodynamics, Phys. Rev. Lett. 106, 042301 (2011).

[40] J.-F. Paquet, C. Shen, G. S. Denicol, M. Luzum, B. Schenke, S. Jeon, and C. Gale, Production of photons in relativistic heavy-ion collisions, Phys. Rev. C 93, 044906 (2016).

[41] http://www.physics.mcgill.ca/music/.

[42] J. E. Bernhard, Ph.D. thesis, Duke University, 2018, arXiv: 1804.06469.

[43] https://github.com/j-f-paquet/eos $\$ maker.

[44] F. Cooper, G. Frye, and E. Schonberg, Landau's hydrodynamic model of particle production and electron positron annihilation into hadrons, Phys. Rev. D 11, 192 (1975).

[45] M. McNelis, D. Everett, and U. Heinz, Particlization in fluid dynamical simulations of heavy-ion collisions: The i S3D module, Comput. Phys. Commun. 258, 107604 (2021).

[46] https://github.com/derekeverett/iS3D.
[47] J. Weil et al., Particle production and equilibrium properties within a new hadron transport approach for heavy-ion collisions, Phys. Rev. C 94, 054905 (2016).

[48] https://github.com/smash-transport/smash.

[49] D. Oliinychenko, V. Steinberg, J. Weil, M. Kretz, H. Elfner (Petersen), J. Staudenmaier, S. Ryu, A. Schfer, J. Rothermel, J. Mohs, F. Li, L. Pang, D. Mitrovic, A. Goldschmidt, L. Geiger, L. Prinz, J.-B. Rose, and J. Hammelmann, smashtransport/smash: Smash-1.6 (2019), https://zenodo.org/ record/3485108\#. YL1EnOspBsM.

[50] G. Başar and D. Teaney, Scaling relation between pA and AA collisions, Phys. Rev. C 90, 054903 (2014).

[51] D. Molnar and Z. Wolff, Self-consistent conversion of a viscous fluid to particles, Phys. Rev. C 95, 024903 (2017).

[52] H. Grad, On the kinetic theory of rarefied gases, Commun. Pure Appl. Math. 2, 331 (1949).

[53] W. Israel, Nonstationary irreversible thermodynamics: A causal relativistic theory, Ann. Phys. (N.Y.) 100, 310 (1976).

[54] W. Israel and J. M. Stewart, Transient relativistic thermodynamics and kinetic theory, Ann. Phys. (N.Y.) 118, 341 (1979).

[55] A. Monnai and T. Hirano, Effects of bulk viscosity at freezeout, Phys. Rev. C 80, 054906 (2009).

[56] G. S. Denicol, H. Niemi, E. Molnar, and D. H. Rischke, Derivation of transient relativistic fluid dynamics from the Boltzmann equation, Phys. Rev. D 85, 114047 (2012); Erratum, Phys. Rev. D 91, 039902 (2015).

[57] S. Chapman, T. G. Cowling, D. Burnett, and C. Cercignani, The Mathematical Theory of Non-uniform Gases: An Account of the Kinetic Theory of Viscosity, Thermal Conduction and Diffusion in Gases (Cambridge University Press, Cambridge, England, 1990).

[58] J. Anderson and H. R. Witting, A relativistic relaxation-time model for the Boltzmann equation, Physica (Amsterdam) 74, 466 (1974).

[59] S. Pratt and G. Torrieri, Coupling relativistic viscous hydrodynamics to Boltzmann descriptions, Phys. Rev. C 82, 044901 (2010).

[60] B. Abelev et al. (ALICE Collaboration), Centrality dependence of $\pi, \mathrm{K}, \mathrm{p}$ production in $\mathrm{Pb}-\mathrm{Pb}$ collisions at $\sqrt{s_{N N}}=2.76 \mathrm{TeV}$, Phys. Rev. C 88, 044910 (2013).

[61] K. Aamodt et al. (ALICE Collaboration), Centrality Dependence of the Charged-Particle Multiplicity Density at Mid-Rapidity in $\mathrm{Pb}-\mathrm{Pb}$ Collisions at $\sqrt{s_{N N}}=2.76 \mathrm{TeV}$, Phys. Rev. Lett. 106, 032301 (2011).

[62] B. B. Abelev et al. (ALICE Collaboration), Event-by-event mean $p_{\mathrm{T}}$ fluctuations in $\mathrm{pp}$ and $\mathrm{Pb}-\mathrm{Pb}$ collisions at the $\mathrm{LHC}$, Eur. Phys. J. C 74, 3077 (2014).

[63] J. Adam et al. (ALICE Collaboration), Measurement of transverse energy at midrapidity in $\mathrm{Pb}-\mathrm{Pb}$ collisions at $\sqrt{s_{\mathrm{NN}}}=2.76 \mathrm{TeV}$, Phys. Rev. C 94, 034903 (2016).

[64] K. Aamodt et al. (ALICE Collaboration), Higher Harmonic Anisotropic Flow Measurements of Charged Particles in $\mathrm{Pb}-\mathrm{Pb}$ Collisions at $\sqrt{s_{N N}}=2.76 \mathrm{TeV}$, Phys. Rev. Lett. 107, 032301 (2011).

[65] B. I. Abelev et al. (STAR Collaboration), Systematic measurements of identified particle spectra in $p p, d^{+} \mathrm{Au}$ and 
$\mathrm{Au}+\mathrm{Au}$ collisions from STAR, Phys. Rev. C 79, 034909 (2009).

[66] J. Adams et al. (STAR Collaboration), Azimuthal anisotropy in $\mathrm{Au}+\mathrm{Au}$ collisions at $\sqrt{s_{N N}}=200-\mathrm{GeV}$, Phys. Rev. C 72, 014904 (2005).

[67] L. Adamczyk et al. (STAR Collaboration), Third harmonic flow of charged particles in $\mathrm{Au}+\mathrm{Au}$ collisions at $\sqrt{s_{N N}}=200 \mathrm{GeV}$, Phys. Rev. C 88, 014904 (2013).

[68] Systematic uncertainties can in principle be non-Gaussian, but generalizing this assumption remains a challenge.

[69] A. R. Liddle, Information criteria for astrophysical model selection, Mon. Not. R. Astron. Soc. 377, L74 (2007).

[70] For simplicity and in accord with the current analysis, all models are assumed to share a common set of parameters and priors.

[71] S. Kullback and R. A. Leibler, On information and sufficiency, Ann. Math. Stat. 22, 79 (1951).

[72] J. B. Rose, J. M. Torres-Rincon, A. Schafer, D. R. Oliinychenko, and H. Petersen, Shear viscosity of a hadron gas and influence of resonance lifetimes on relaxation time, Phys. Rev. C 97, 055204 (2018).

[73] J. B. Rose, J. M. Torres-Rincon, and H. Elfner, Inclusive and effective bulk viscosities in the hadron gas, J. Phys. G $\mathbf{4 8}$, 015005 (2021).

[74] E. Lu and G. D. Moore, The bulk viscosity of a pion gas, Phys. Rev. C 83, 044901 (2011).

[75] F. Karsch, D. Kharzeev, and K. Tuchin, Universal properties of bulk viscosity near the QCD phase transition, Phys. Lett. B 663, 217 (2008).

[76] J. Noronha-Hostler, J. Noronha, and C. Greiner, Transport Coefficients of Hadronic Matter near $T_{c}$, Phys. Rev. Lett. 103, 172302 (2009).

[77] P. B. Arnold, C. Dogan, and G. D. Moore, The bulk viscosity of high-temperature QCD, Phys. Rev. D 74, 085021 (2006).

[78] http://www.tacc.utexas.edu.

[79] Ohio supercomputer center (1987). 